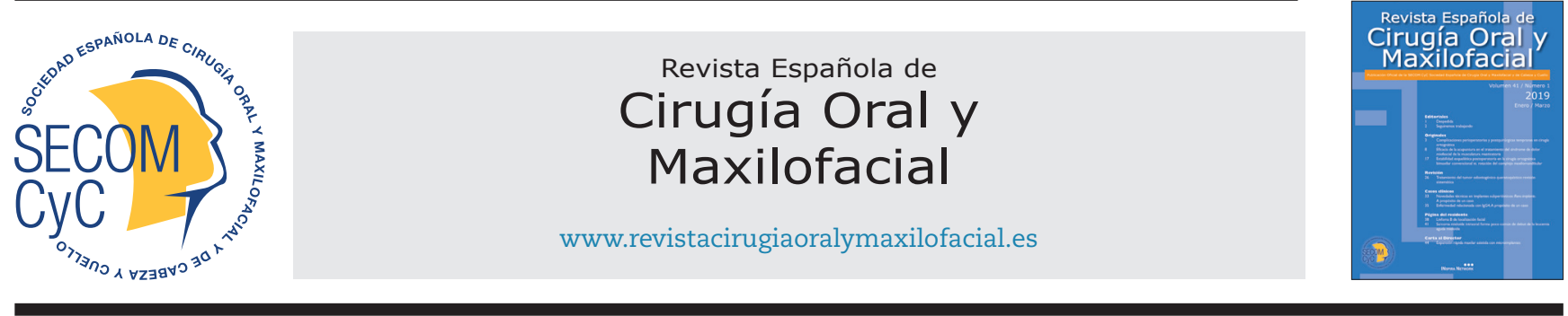

\title{
Original
}

\section{Estabilidad esquelética postoperatoria en la cirugía ortognática bimaxilar convencional us. rotación del complejo maxilomandibular}

\author{
Félix José Amarista Rojas ${ }^{a}$, Néstor Ricardo González Marín ${ }^{b}$, Omar Alejandro Vega Lagos ${ }^{a}$, \\ Sergio Mauricio Castellanos García ${ }^{a}$ y Camilo Alberto Eslava Jácome ${ }^{a}$
}

aServicio de Cirugía Oral y Maxilofacial y bServicio de Otorrinolaringología. Hospital Militar Central de Bogotá. Universidad Militar Nueva Granada. Bogotá, Colombia

\section{INFORMACIÓN DEL ARTÍCULO}

Historia del artículo:

Recibido el 05 de octubre de 2017

Aceptado el 10 de junio de 2018

\section{Palabras clave:}

Cirugía ortognática, alteración plano oclusal, rotación antihoraria, rotación horaria.

\section{R E S U M E N}

Introducción: La estabilidad esquelética postoperatoria en cirugía ortognática ha sido objeto de evaluación durante el paso del tiempo. Diversos autores han reportado que se produce una disminución de la misma en diversos movimientos de los maxilares en ocasiones utilizados en casos de rotación del complejo maxilomandibular. El objetivo del presente trabajo fue comparar la estabilidad esquelética postoperatoria de la cirugía ortognática bimaxilar convencional us. cirugía ortognática bimaxilar con rotación del complejo maxilomandibular en pacientes sometidos a cirugía ortognática en el Servicio de Cirugía Oral y Maxilofacial del Hospital Militar Central de Bogotá, entre enero de 2012 hasta julio de 2016.

Materiales y métodos: Se trata de un estudio retrospectivo. Se llevó a cabo análisis cefalométrico para comparar la estabilidad esquelética entre los pacientes de cirugía ortognática bimaxilar convencional us. los pacientes de cirugía ortognática bimaxilar con rotación del complejo maxilomandibular durante tres momentos: preoperatorio (T1), postoperatorio inmediato (T2), postoperatorio al menos de seis meses (T3).

Resultados: Se obtuvo una cohorte de 45 pacientes sometidos a cirugía ortognática bimaxilar convencional o cirugía ortognática bimaxilar con rotación del complejo maxilomandibular. La mayoría de las medidas realizadas mostraron que no existe diferencia significativa de estabilidad a largo plazo en ambos grupos.

Conclusiones: La cirugía ortognática con rotación del complejo maxilomandibular, tanto en sentido horario como antihorario, es un procedimiento estable cuando se utiliza fijación interna rígida, cuando se está en presencia de articulacion temporomandibular (ATM) sanas y cuando la rotación se lleva a cabo en un punto a través del cóndilo mandibular.

\footnotetext{
${ }^{*}$ Autor para correspondencia

Correo electrónico: felixjoseamarista@hotmail.com (Félix José Amarista Rojas).

https://doi.org/10.20986/recom.2019.1031/2019
}

1130-0558/@ 2019 SECOM. Publicado por Inspira Network. Este es un artículo Open Access bajo la licencia CC BY-NC-ND (http:// creativecommons.org/licenses/by-nc-nd/4.0/). 


\section{Keywords:}

Orthognathic surgery, occlusal plane alteration, counterclockwise rotation, clockwise rotation.

\section{A B S T R A C T}

Introduction: Postoperative skeletal stability in orthognathic surgery has been evaluated over time, several authors have reported a decrease of it in movements of the jaws occasionally used in cases of alteration of the maxillo-mandibular complex. The aim of the present study was to compare the postoperative skeletal stability of conventional bimaxillary orthognathic surgery versus bimaxillary orthognathic surgery with rotation of the maxillo-mandibular complex in patients undergoing orthognathic surgery at the Oral and Maxillofacial Surgery Service of the Central Military Hospital of Bogotá between January 2012 until July 2016.

Materials and methods: A retrospective study was conducted. We performed a cephalometric analysis to compare the skeletal stability between patients with conventional bimaxillary orthognathic surgery versus bimaxillary orthognathic surgery patients with maxillomandibular alteration during 3 moments: before surgery (T1), right after the surgery (T2), at least 6 months after surgery (T3).

Results: A cohort of 45 patients undergoing conventional bimaxillary orthognathic surgery or bimaxilar orthognathic surgery with rotation of the maxillo-mandibular complex was obtained. Most of the measurements showed that there is no significant difference in long-term stability in both groups.

Conclusions: Orthognathic surgery with rotation of the maxillo-mandibular complex both clockwise and counter-clockwise is a stable procedure when rigid internal fixation is used, when the temporomandibular joints (TMJs) are healthy and stable and when rotation is performed at a point through the mandibular condyle.

\section{Introducción}

La cirugía ortognática bimaxilar con rotación del complejo maxilomandibular es considerada una técnica efectiva que permite la modificación del ángulo de planos oclusales hiperdivergentes o hipodivergentes, así como lograr resultados estéticos ideales en casos de anomalías dentofaciales (ADF), en donde no existen discrepancias antero posteriores suficientes para lograr resultados adecuados.

La estabilidad esquelética postoperatoria en cirugía ortognática ha sido objeto de evaluación durante el paso del tiempo. Actualmente el uso de fijación interna rígida ha traído como consecuencia resultados estables a largo plazo, sin embargo, diversos autores han reportado que se produce una disminución en la estabilidad esquelética postoperatoria en diversos movimientos de los maxilares en ocasiones utilizados en casos de rotación del complejo maxilomandibular ${ }^{1,2}$.

Como consecuencia de lo anteriormente descrito, surge la necesidad de comparar la estabilidad esquelética postoperatoria de la cirugía ortognática bimaxilar convencional us. cirugía ortognática bimaxilar con rotación del complejo maxilomandibular en pacientes sometidos a cirugía ortognática en el Servicio de Cirugía Oral y Maxilofacial del Hospital Militar Central de Bogotá.

\section{Materiales y métodos}

El presente estudio evaluó 45 pacientes sometidos a cirugía ortognática bimaxilar consistente en osteotomía Le Fort I convencional o alta dependiendo de las necesidades de los pacientes y osteotomía sagital de rama mandibular bilateral (OSRMB) mediante la modificación de Hunsuck ${ }^{3}$; en los casos de rotaciones del complejo maxilomandibular se llevó a cabo desinserción de la cincha pterigomaseterina.

La osteotomía Le Fort I fue estabilizada con miniplacas en L sistema 2.0 y tornillos monocorticales, dos en pilar nasomaxilar bilateral y dos en pilar maxilomandibular y las OSRMB mediante fijación interna rígida con miniplacas y tornillos o tornillos bicorticales.

En los casos de rotación del complejo maxilomandibular con descensos anteriores o posteriores del maxilar, se colocó injerto óseo cortical de interposición, ya sea autólogo en casos de disponibilidad ósea posterior a mentoplastias concomitantes de reducción vertical o aloinjertos.

\section{Tipo y diseño general del estudio}

Se llevó a cabo un estudio retrospectivo con el fin de comparar la estabilidad esquelética postoperatoria de la cirugía ortognática bimaxilar convencional us. cirugía ortognática bimaxilar con rotación del complejo maxilomandibular en pacientes sometidos a cirugía ortognática en el Servicio de Cirugía Oral y Maxilofacial del Hospital Militar Central de Bogotá, entre enero de 2012 hasta julio de 2016.

\section{Selección y tamaño de la muestra}

Muestreo no probabilístico por conveniencia. Se incluyeron todos los pacientes que quiesieran participar, que 
hubieran sido sometidos a cirugía ortognática bimaxilar en el periodo establecido y que cumplieran con los criterios de inclusión.

\section{Criterios de inclusión}

- Pacientes sometidos a cirugía ortognática bimaxilar convencional o rotación del complejo maxilomandibular con fijación interna con material de osteosíntesis tipo placas y tornillos.

- Disponibilidad de radiografías cefálicas laterales estandarizadas de calidad y resolución adecuada tomadas de acuerdo con protocolo, tomadas en tres tiempos (preoperatorio, postoperatorio inmediato, postoperatorio seis meses).

- Paciente con potencial de crecimiento mínimo (femenino mayor de 15 años y masculino mayor de 17 años).

\section{Métodos para evaluación}

Se llevó a cabo un análisis cefalométrico para comparar la estabilidad esquelética entre los pacientes de cirugía ortognática bimaxilar convencional us. los pacientes de cirugía ortognática bimaxilar con rotación del complejo maxilomandibular.

La medición principal se realizó en base a las radiografías cefálicas laterales de perfil tomadas de acuerdo a protocolo de seguimiento clínico. Estas radiografías se denominaron preoperatorio (T1), postoperatorio inmediato (T2), postoperatorio al menos de seis meses (T3). Se utilizaron las respectivas radiografías para realizar un análisis cefalométrico en hojas de acetato, las cuales fueron trazadas por el mismo examinador de forma aleatoria y sin el conocimiento de la identificación del paciente.

Se realizó la marcación de los puntos cefalométricos silla $(\mathrm{S})$, nasion $(\mathrm{N})$, punto $\mathrm{A}$, punto $\mathrm{B}$, espina nasal posterior (Enp), gonion (G), mentón (M) y pogonion (Pg) en T1, T2 y T3. Para evaluar los cambios óseos quirúrgicos y la estabilidad a largo plazo se construyeron los siguientes planos: la base craneal anterior o S-N, plano horizontal verdadero $\left(7^{\circ}\right.$ del plano $\mathrm{S}-\mathrm{N}$ a través $\mathrm{S})$, el plano oclusal (PO) y el plano mandibular (PM) (gonionmentón). El plano horizontal verdadero se utilizó como el eje Y para mediciones verticales mientras que el eje X se construyó a partir de una línea perpendicular al eje Y a través de silla y se utilizó para medir los cambios horizontales. Para evaluar los cambios antero-posteriores del maxilar y la mandíbula, se llevó a cabo la medida de la distancia lineal en milímetros desde el plano vertical (Eje X) al punto A (AX), al punto B (BX), a la Enp (EnpX), al pogonion (PgX) y al mentón (MX) mientras que los cambios verticales se evaluaron mediante la medición de la distancia lineal en milímetros desde el plano horizontal (Eje Y) al punto A (AY), al punto B (BY), a la Enp (EnpY), al pogonion (PgY) y al mentón (MY). El PO se construyó trazando una línea a través de las cúspides de los primeros molares y el borde incisal.

Se realizó la medida de los ángulos SNA, SNB y SNPg y el cambio en el ángulo del PO y el PM se midió en relación con el plano horizontal verdadero construido.
Los casos con cambio del PO de más de $+2^{\circ}$ se consideraron como rotación horaria del complejo maxilomandibular $(\mathrm{RH})$, mientras que los casos con cambio del PO de $-2^{\circ}$ y más negativos se consideraron como rotación antihoraria del complejo maxilomandibular (RAH). Todos los casos con cambios del PO entre $+2^{\circ}$ y $-2^{\circ}$ se consideraron como cirugía ortognática convencional (TC).

\section{Análisis estadístico}

La descripción de las variables se hizo mediante medidas de tendencia central (media, mediana y moda, según su distribución) y dispersión (desviación estándar, rango intercuartil) para las variables cuantitativas; se realizó pruebas de normalidad de Kolmogorov-Smirnov para evaluar la distribución de los datos con respecto a la normal ( $p>0,05$ para este estudio), para las variables categóricas se describió frecuencias a través de proporciones.

Para el contraste de hipótesis, se usaron pruebas de estadística paramétrica dada la distribución normal de los datos (K-S p > 0,05), se utilizó la prueba t de Student para muestras relacionadas con el objetivo de identificar diferencias de T2-T1 y T3-T2 entre el mismo grupo en dos momentos dierentes del seguimiento, identificando así la recidiva u otros movimientos posteriores al POP inmediato. De igual manera se usó la prueba t de Student para muestras independientes con el propósito de contrastar la hipótesis de diferencia en la estabilidad entre los dos grupos en estudio. Este análisis se llevó a cabo mediante el programa IBM SPSS versión 21.0 para OS, Chicago, IL, EE. UU.

\section{Resultados}

Se reclutaron 65 pacientes del servicio de Cirugía Oral y Maxilofacial del Hospital Militar Central con ADF sometidos a cirugía ortognática bimaxilar en el Servicio de Cirugía Oral y Maxilofacial desde el 1 de enero de 2012 hasta el 31 de julio de 2016. Veinte pacientes con criterios de exclusión, por lo que se obtuvo una cohorte de 45 pacientes sometidos a cirugía ortognática bimaxilar convencional o cirugía ortognática bimaxilar con rotación del complejo maxilomandibular.

Las características clínicas y demográficas de los pacientes incluidos en la cohorte en estudio se describen en la Tabla I. Se evidenció una media de edad de 24,8 años, predominio del género femenino en un $62,2 \%$ y ADF clase III con un 51,1 \%. El tiempo de seguimiento posoperatorio promedio fue de 20 meses (DE \pm 13,3).

Los pacientes fueron asignados a cada grupo de observación según si fueron sometidos a cirugía ortognática bimaxilar convencional en el Grupo I ( $n=15$ ) o si se llevó a cabo cirugía ortognática con rotación del complejo maxilomandibular, la cual consistía en variación del plano oclusal (PO) con respecto a la verdadera horizontal $(\mathrm{VH})$ en +2 o -2 grados, Grupo II $(n=30)$. Se puede observar una media de edad de 25,32 años en el Grupo I y de 24,58 años en el Grupo II. Con respecto al tipo de ADF, se observó un predominio de ADF clase III en el Grupo I (73,3 \%), sin embargo, en el grupo II se evidenció un predominio de ADF clase II (56,6\%). 
Tabla I. Características clínicas y demográficas de los pacientes sometidos a cirugía ortognática bimaxilar con rotación del complejo maxilomandibular us. cirugía ortognática bimaxilar convencional en el Hospital Militar Central

Cirugía ortognática bimaxilar convencional

(GI)
Cirugía ortognática bimaxilar con rotación del complejo maxilomandibular (GII)

\begin{tabular}{lcccccccccc}
\hline & Media & DE & mín. & máx. & Media & DE & mín. & máx. & Media & DE \\
\hline $\begin{array}{l}\text { Edad } \\
\text { (años) }\end{array}$ & 25,32 & 8,39 & 16,24 & 37,48 & 24,58 & 6,82 & 15,66 & 43,75 & 24,8 & 7,3
\end{tabular}

Seguimiento POP

(meses)

$19,8 \quad 13,1$

$6 \quad 49$

20,1

13,6

6

56

20

13,3

\section{Género}

Femenino

$(\mathrm{n}=15)$

$100 \%$

$(\mathrm{n}=30)$

$100 \%$

$\mathrm{n}$

$\%$

Masculino

9

9
6

60

19

11

63,3

36,7

28

28
17

62,2

ADF

Clase I

Clase II

Clase III

$\begin{array}{ll}2 & 13,3 \\ 2 & 13,3 \\ 11 & 73,3\end{array}$

13,3

13,3

$1 \quad 3,3$

3,3

56,7

12

40

GI: Grupo I. GII: Grupo Ii. DE: desviación estándar. mín.: mínimo. máx.: máximo.

Con respecto al tipo de material de osteosíntesis utilizado en cada grupo, la osteotomía Le Fort I fue estabilizada en el $100 \%$ de los casos mediante cuatro miniplacas en L sistema 2.0 con tornillos bicorticales. La OSRMB en el grupo de cirugía ortognática convencional se estabilizó mediante tornillos bicorticales en el 60,7 \% de los casos, una miniplaca con tornillos monocorticales (25\%), una miniplaca con tornillos monocorticales + tornillo bicortical adicional (10,7 \%) y dos miniplacas con tornillos monocorticales (3,6 \%). Por otro lado, la OSRMB del grupo de cirugía ortognática con rotación del complejo maxilomandibular fue estabilizada mediante tornillos bicorticales en el 47,1 \% de los casos, una miniplaca con tornillos monocorticales (41,2\%), una miniplaca con tornillos monocorticales + tornillo bicortical adicional $(15,9 \%)$ y dos miniplacas con tornillos monocorticales (5,9\%).

\section{Grupo I: cirugía ortognática bimaxilar convencional}

Este grupo fue conformado por 15 pacientes. El promedio del cambio de plano oclusal fue de $-0,37^{\circ}$ (POVH T2-T1) y el cambio del plano mandibular fue de $-2,30^{\circ}$ (MPVH T2-T1). El maxilar avanzó en promedio 2,97 mm (AX T2-T1), sin embargo, el punto B retrocedió en promedio 3,67 mm (BX T2-T1) y la espina nasal posterior presentó un avance de 2,63 mm (EnpX T2-T1). Con respecto a las medidas de referencia al mentón, el pogonion tuvo un retroceso de 0,63 mm (PgX T2-T1) y el punto $\mathrm{M}$ retrocedió en promedio 0,40 mm (MX T2-T1) (Tabla II).

El punto A tuvo un ascenso promedio de 0,07 $\mathrm{mm}$ (AY T2-T1), el punto B de 0,57 mm (BY T2-T1) y la espina nasal posterior no presentó cambios (EnpY T2-T1). Por otro lado, el pogonion tuvo un ascenso de 0,90 mm (PgY T2-T1) y el punto M de 1,10 mm (MY T2-T1) (Tabla II).

En referencia a las medidas de ángulos faciales en SNA tuvo un incremento promedio de $2,47^{\circ}$ (SNA T2-T1), el SNB dismi- nuyó en 2,09 (SNB T2-T1) y el SNPg una disminución de 0,27 (SNPg T2-T1).

En el postoperatorio (T3-T2), el maxilar se movió posteriormente 0,03 $\mathrm{mm}$ promedio, la mandíbula en punto B avanzó 2,77 $\mathrm{mm}$ y la espina nasal posterior presentó una recidiva de 0,97 mm. El pogonion avanzó de 2,60 mm y el punto $\mathrm{M}$ 2,43 $\mathrm{mm}$.

Desde el punto de vista vertical el punto A descendió 0,73 mm, el punto B 0,50 mm y la Enp 1,00 mm. El Pg avanzó 1,60 $\mathrm{mm}$ y el punto $\mathrm{M} 1,03 \mathrm{~mm}$.

El ángulo SNA disminuyó $1,23^{\circ}$ en el postoperatorio, SNB aumentó $0,80^{\circ}$ y SNPg $0,47^{\circ}$. El plano oclusal con respecto a la verdadera horizontal presentó una disminución de $0,27^{\circ}$ y el plano mandibular aumentó $0,40^{\circ}$.

\section{Grupo II: Cirugía ortognática bimaxilar con rotación del complejo maxilomandibular}

A este grupo correspondieron 30 pacientes; el promedio de cambio de plano oclusal fue de $-5,18^{\circ}$ (POVH T2-T1), lo que indica rotación antihoraria del complejo maxilomandibular; el cambio del plano mandibular fue de $-5,90^{\circ}$ (MPVH T2-T1). El punto A presentó un avance promedio de 3,35 mm (AX T2-T1), el punto B de 5,48 mm (BX T2-T1) y la Enp de 2,77 mm (EnpX T2-T1). Con respecto a las medidas de referencia al mentón, el pogonion tuvo un avance de 10,23 mm (PgX T2-T1) y el punto $\mathrm{M}$ retrocedió en promedio 10,98 mm (MX T2-T1) (Tabla III).

El punto A tuvo un ascenso promedio de $3,93 \mathrm{~mm}$ (AY T2-T1), el punto B de 5,50 mm (BY T2-T1) y la Enp de 0,32 mm (EnpY T2-T1). Por otro lado, el pogonion tuvo un ascenso de 3,52 (PgY T2-T1) y el punto M de 3,03 mm (MY T2-T1). El movimiento superior del maxilar a nivel del punto A deja en evidencia la rotación antihoraria del complejo maxilomandibular, sin embargo, algunos casos requirieron adicio- 
Tabla II. Estabilidad esquelética en el postoperatorio inmediato y comparación con la estabilidad en el postoperatorio mínimo de seis meses de los pacientes sometidos a cirugía ortognática bimaxilar convencional

\begin{tabular}{|c|c|c|c|c|c|c|c|c|c|c|c|}
\hline \multirow{2}{*}{ Cefalometría } & \multicolumn{11}{|c|}{ Grupo I $(\mathrm{n}=15)$} \\
\hline & T1 & SD & T2 & SD & T3 & SD & T2-T1 & $\mathrm{SD}$ & T3-T2 & SD & $\mathrm{p}^{*}$ \\
\hline \multicolumn{12}{|l|}{$\begin{array}{l}\text { Milímetros } \\
\text { (mm) }\end{array}$} \\
\hline $\mathrm{AX}$ & 70,30 & 7,29 & 73,52 & 7,14 & 73,54 & 7,54 & 2,97 & 4,61 & $-0,03$ & 3,30 & 0,97 \\
\hline $\mathrm{BX}$ & 67,13 & 13,98 & 69,57 & 9,63 & 70,84 & 10,94 & $-3,67$ & 4,89 & 2,77 & 3,98 & 0,02 \\
\hline EnpX & 22,74 & 4,92 & 25,47 & 5,32 & 24,60 & 5,26 & 2,63 & 2,46 & $-0,97$ & 2,03 & 0,09 \\
\hline $\operatorname{PgX}$ & 68,17 & 15,29 & 74,78 & 10,20 & 75,44 & 11,82 & $-0,63$ & 4,27 & 2,60 & 4,06 & 0,03 \\
\hline MX & 61,61 & 15,51 & 68,80 & 10,45 & 69,40 & 12,02 & $-0,40$ & 3,93 & 2,43 & 4,16 & 0,04 \\
\hline AY & 51,16 & 4,77 & 48,51 & 5,47 & 49,85 & 4,75 & $-0,07$ & 2,80 & 0,73 & 2,60 & 0,29 \\
\hline BY & 98,34 & 7,85 & 94,49 & 7,32 & 95,92 & 7,00 & $-0,57$ & 5,30 & 0,50 & 3,36 & 0,57 \\
\hline EnpY & 45,94 & 3,68 & 45,73 & 4,25 & 46,83 & 3,93 & 0,00 & 2,95 & 1,00 & 2,35 & 0,12 \\
\hline PgY & 110,72 & 7,47 & 108,08 & 7,84 & 110,41 & 6,91 & $-0,90$ & 6,41 & 1,60 & 4,77 & 0,22 \\
\hline MY & 118,42 & 8,12 & 116,03 & 8,10 & 117,94 & 7,05 & $-1,10$ & 6,34 & 1,03 & 5,10 & 0,45 \\
\hline \multicolumn{12}{|l|}{ Grados $\left({ }^{\circ}\right)$} \\
\hline SNA & 83,19 & 4,82 & 86,16 & 4,96 & 85,30 & 4,75 & 2,47 & 3,01 & $-1,23$ & 1,39 & 0,00 \\
\hline SNB & 81,11 & 6,71 & 82,26 & 4,59 & 82,28 & 4,94 & $-2,09$ & 2,50 & 0,80 & 1,39 & 0,04 \\
\hline SNPg & 81,69 & 6,78 & 84,81 & 4,47 & 84,53 & 4,81 & $-0,27$ & 2,22 & 0,47 & 1,30 & 0,19 \\
\hline POVH & 11,52 & 6,87 & 7,94 & 4,73 & 8,28 & 5,43 & $-0,37$ & 1,48 & $-0,27$ & 1,64 & 0,54 \\
\hline MPVH & 30,16 & 7,31 & 25,46 & 5,93 & 26,42 & 6,09 & $-2,30$ & 2,85 & 0,40 & 1,31 & 0,26 \\
\hline
\end{tabular}

*Prueba t-Student para muestras relacionadas.

Tabla III. Estabilidad esquelética en el postoperatorio inmediato y comparación de la estabilidad en el postoperatorio mínimo de seis meses en los pacientes sometidos a cirugía ortognática bimaxilar con rotación del complejo maxilo-mandibular

\begin{tabular}{|c|c|c|c|c|c|c|c|c|c|c|c|}
\hline \multirow{2}{*}{ Cefalometría } & \multicolumn{11}{|c|}{ Grupo II $(\mathrm{n}=30)$} \\
\hline & $\mathrm{T} 1$ & SD & $\mathrm{T} 2$ & SD & T3 & SD & $\mathrm{T} 2-\mathrm{T} 1$ & SD & T3-T2 & SD & $\mathrm{p}^{*}$ \\
\hline \multicolumn{12}{|l|}{$\begin{array}{l}\text { Milímetros } \\
(\mathrm{mm})\end{array}$} \\
\hline $\mathrm{AX}$ & 70,13 & 8,21 & 73,48 & 8,28 & 73,53 & 8,71 & 3,35 & 3,24 & 0,05 & 1,69 & 0,87 \\
\hline $\mathrm{BX}$ & 63,97 & 14,70 & 69,45 & 10,79 & 69,98 & 12,17 & 5,48 & 8,51 & 0,53 & 2,95 & 0,33 \\
\hline EnpX & 22,98 & 5,89 & 25,75 & 6,17 & 24,93 & 6,19 & 2,77 & 2,88 & $-0,82$ & 2,28 & 0,06 \\
\hline $\mathrm{PgX}$ & 64,58 & 16,09 & 74,82 & 11,09 & 74,52 & 12,78 & 10,23 & 10,06 & $-0,30$ & 3,34 & 0,63 \\
\hline $\mathrm{MX}$ & 57,87 & 16,20 & 68,85 & 11,18 & 68,53 & 12,72 & 10,98 & 9,84 & $-0,32$ & 3,47 & 0,62 \\
\hline AY & 51,70 & 4,91 & 47,77 & 5,84 & 49,41 & 5,48 & $-3,93$ & 3,93 & 1,65 & 2,50 & 0,00 \\
\hline BY & 98,87 & 7,46 & 93,37 & 7,44 & 95,27 & 7,41 & $-5,50$ & 6,45 & 1,90 & 3,24 & 0,00 \\
\hline EnpY & 46,00 & 3,68 & 45,68 & 4,47 & 46,83 & 4,52 & $-0,32$ & 2,59 & 1,15 & 1,45 & 0,00 \\
\hline PgY & 110,58 & 6,76 & 107,07 & 8,20 & 109,77 & 7,60 & $-3,52$ & 5,90 & 2,70 & 3,56 & 0,00 \\
\hline MY & 118,18 & 7,88 & 115,15 & 8,54 & 117,50 & 7,94 & $-3,03$ & 6,39 & 2,35 & 3,56 & 0,00 \\
\hline \multicolumn{12}{|l|}{ Grados $\left({ }^{\circ}\right)$} \\
\hline SNA & 83,15 & 5,26 & 86,37 & 5,53 & 85,70 & 5,18 & 3,22 & 2,20 & $-0,67$ & 2,23 & 0,11 \\
\hline SNB & 79,58 & 6,85 & 82,35 & 5,00 & 81,98 & 5,31 & 2,77 & 4,40 & $-0,37$ & 1,60 & 0,22 \\
\hline SNPg & 80,12 & 6,95 & 84,93 & 4,58 & 84,28 & 4,96 & 4,82 & 4,65 & $-0,65$ & 1,56 & 0,03 \\
\hline POVH & 13,02 & 7,50 & 7,83 & 4,94 & 8,47 & 5,87 & $-5,18$ & 5,04 & 0,63 & 2,72 & 0,21 \\
\hline MPVH & 31,10 & 8,32 & 25,2 & 6,54 & 26,45 & 6,79 & $-5,90$ & 4,23 & 1,25 & 1,67 & 0,00 \\
\hline
\end{tabular}

${ }^{*}$ Prueba t-Student para muestras relacionadas. 
nalmente de descensos posteriores para aumentar la rotación del complejo maxilomandibular (Tabla III).

En referencia a las medidas de ángulos faciales en SNA tuvo un incremento promedio de $3,22^{\circ}$ (SNA T2-T1), el SNB aumento $2,77^{\circ}$ (SNB T2-T1) y el SNPg una disminución de 4,82 ${ }^{\circ}$ (SNPg T2-T1). (Tabla III).

En el postoperatorio (T3-T2), el maxilar avanzó 0,05 mm promedio, la mandíbula en punto B avanzó 0,53 mm y la Enp presentó una recidiva de $0,82 \mathrm{~mm}$. El pogonion tuvo una recidiva de 0,30 $\mathrm{mm}$ y el punto $\mathrm{M} 0,32 \mathrm{~mm}$.

Desde el punto de vista vertical, el punto A descendió 1,65 mm, el punto B 1,90 mm y la Enp 1,15 mm. El Pg avanzó 2,70 $\mathrm{mm}$ y el punto $\mathrm{M} 2,35 \mathrm{~mm}$.

El ángulo SNA aumentó $2,23^{\circ}$ en el postoperatorio, SNB aumentó $1,60^{\circ}$ y SNPg $1,56^{\circ}$. El plano oclusal con respecto a la verdadera horizontal presentó un aumento de $0,63^{\circ} \mathrm{y}$ el plano mandibular aumentó 2,35 .

Los cambios quirúrgicos a largo plazo fueron comparados entre el grupo de cirugía ortognática bimaxilar convencional us. cirugía ortognática bimaxilar con rotación del complejo maxilomandibular y los resultados fueron tabulados en la Tabla IV.

Tabla IV. Comparación de la estabilidad esquelética postoperatoria de los pacientes sometidos cirugía ortognática bimaxilar convencional us. cirugía ortognática bimaxilar con rotación del complejo maxilomandibular

\begin{tabular}{|c|c|c|c|c|c|}
\hline \multirow{2}{*}{ Cefalometría } & \multicolumn{2}{|c|}{$\begin{array}{c}\text { T3-T2 } \\
\text { GI }(n=15)\end{array}$} & \multicolumn{2}{|c|}{$\begin{array}{c}\text { T3-T2 } \\
\text { GII }(n=30)\end{array}$} & \multirow[t]{2}{*}{$\mathrm{p}^{*}$} \\
\hline & $\begin{array}{l}\text { Dif de } \\
\text { Medias }\end{array}$ & $\mathrm{DE}$ & $\begin{array}{l}\text { Dif de } \\
\text { Medias }\end{array}$ & $\mathrm{DE}$ & \\
\hline \multicolumn{6}{|l|}{$\begin{array}{l}\text { Milímetros } \\
(\mathrm{mm})\end{array}$} \\
\hline $\mathrm{AX}$ & $-0,03$ & 3,30 & 0,05 & 1,69 & 0,93 \\
\hline $\mathrm{BX}$ & 2,77 & 3,98 & 0,53 & 2,95 & 0,04 \\
\hline EnpX & $-0,97$ & 2,03 & $-0,82$ & 2,28 & 0,83 \\
\hline $\operatorname{PgX}$ & 2,60 & 4,06 & $-0,30$ & 3,34 & 0,01 \\
\hline MX & 2,43 & 4,16 & $-0,32$ & 3,47 & 0,02 \\
\hline AY & 0,73 & 2,60 & 1,65 & 2,50 & 0,26 \\
\hline BY & 0,50 & 3,36 & 1,90 & 3,24 & 0,18 \\
\hline EnpY & 1,00 & 2,35 & 1,15 & 1,45 & 0,79 \\
\hline PgY & 1,60 & 4,77 & 2,70 & 3,56 & 0,39 \\
\hline MY & 1,03 & 5,10 & 2,35 & 3,56 & 0,32 \\
\hline \multicolumn{6}{|l|}{ Grados $\left({ }^{\circ}\right)$} \\
\hline SNA & $-1,23$ & 1,39 & $-0,67$ & 2,23 & 0,37 \\
\hline SNB & 0,80 & 1,39 & $-0,37$ & 1,60 & 0,02 \\
\hline SNPg & 0,47 & 1,30 & $-0,65$ & 1,56 & 0,02 \\
\hline $\mathrm{POVH}$ & $-0,27$ & 1,64 & 0,63 & 2,72 & 0,25 \\
\hline MPVH & 0,40 & 1,31 & 1,25 & 1,67 & 0,09 \\
\hline
\end{tabular}

*Prueba T- Student para muestras independientes.

\section{Cambios quirúrgicos y postoperatorios anteroposteriores}

En ambos grupos se llevó a cabo avance del maxilar durante el procedimiento quirúrgico (AX T2-T1) (Figura 1). La comparación de la estabilidad esquelética postoperatoria con respecto al punto A no fue estadísticamente significativa $(p=0,93)$ (Tabla IV). El punto B presentó un avance (Figura 1) sustancialmente mayor en el grupo II (BX T2-T1 = 5,48 mm) (Tabla II), esto como consecuencia de la rotación antihoraria, sin embargo, presentó una cambio postoperatorio mayor en el Grupo I con una diferencia estadísticamente significativa $(p=0,04)$ (Tabla IV). La Enp fue avanzada en ambos grupos (EnpX T2-T1) (Figura 1) y la estabilidad esquelética no presentó diferencias significativas (EnpX T3-T2) (Tabla IV).

\section{Cambios quirúrgicos y postoperatorios verticales}

Desde el punto de vista vertical, el punto A y B presentaron un reposicionamiento superior o de ascenso (AY T2-T1) (BY T2-T1) (Figura 2), considerablemente mayor en el Grupo II, esto como consecuencia de la impactación anterior para lograr la

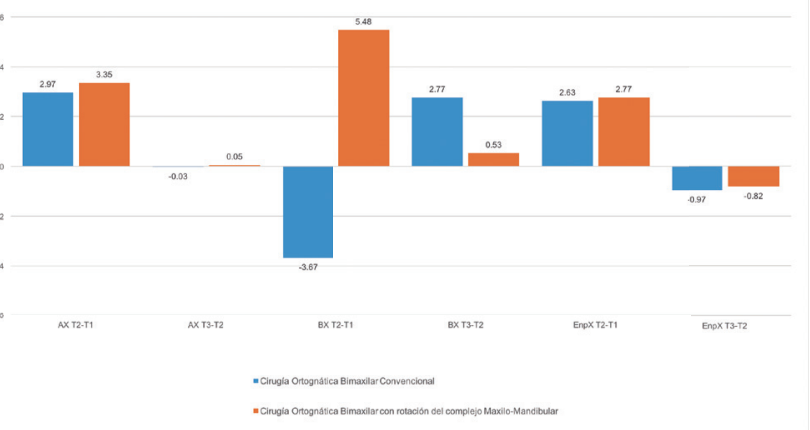

Figura 1. Cambios quirúrgicos y postquirúrgicos anteroposteriores.

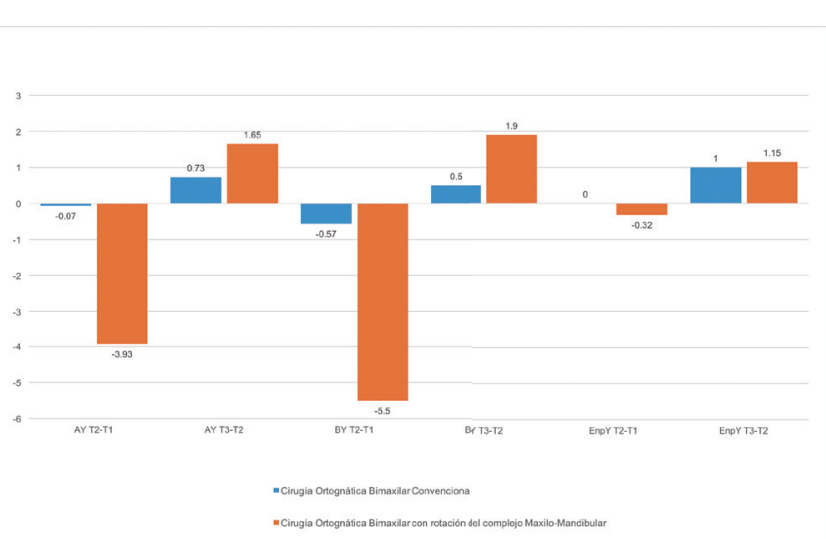

Figura 2. Cambios quirúrgicos y postquirúrgicos verticales. 
rotación antihoraria del complejo maxilomandibular. La Enp no presentó cambios importantes durante el procedimiento quirúrgico (EnpY T2-T1). La comparación en la estabilidad esquelética vertical postoperatoria en todas las medidas no fue estadísticamente significativa (Tabla IV).

\section{Cambios quirúrgicos y postoperatorios de mentoplastia}

El pogonion y el punto $\mathrm{M}$ en el Grupo I presentaron un movimiento de retroceso en la mayoría de los casos. Por otro lado, en el Grupo II se llevó a cabo un avance promedio de 10,23 mm en el Pg (PgX T2-T1) y 10,98 mm en el punto M (MX T2-T1) (Figura 3), esto debido posiblemente a la alta prevalencia de ADF clase III (73,3\%) y ADF clase II (56,6 \%) en cada grupo, respectivamente (Tabla I). En cuanto a la estabilidad esquelética, se produjo mayor cambio postoperatorio en el Grupo I (PgX T3-T2) (MX T3-T2), obteniendo una diferencia estadísticamente significativa $p=0,01$ y $p=0,02$, respectivamente (Tabla IV).

Desde el punto de vista vertical el Pgy el punto M, en ambos grupos tuvieron un movimiento de ascenso (PgY T2-T1) (MY T2-T1), mayor en el grupo II a nivel de $\mathrm{Pg}(3,52 \mathrm{~mm})$ y a nivel de M (3,03 mm) (Figura 3), como consecuencia de la disminución del tercio inferior producida por la rotación antihoraria del complejo maxilomandibular. Sin embargo, no hubo diferencia significativa en cuanto a la estabilidad esquelética entre grupos (PgY T3-T2) (MY T3-T2) (Tabla IV).

\section{Cambios quirúrgicos y postoperatorios de ángulos faciales}

El plano oclusal (POVH) y el plano mandibular (MPVH) en ambos grupos fueron sometidos una rotación antihoraria (Tabla II y III), mayor en el Grupo II como consecuencia de la rotación antihoraria. Los cambios postoperatorios a largo plazo no fueron estadísticamente significativos (Tabla IV).
Se produjo un aumento en grados en el ángulo SNA en ambos grupos (Figura 4) sin cambios postoperatorios significativos (Tabla IV). Por otro lado, el ángulo SNB y SNPg fue disminuido en el grupo I y aumentado en el grupo II (Figura 4) con cambios estadísticamente significativos en ambos $(p=0,02)($ Tabla IV).

\section{Discusión}

Van Sickels refiere que para todos los procedimientos quirúrgicos existe cierto grado de recidiva tanto en la utilización de alambre de osteosíntesis como en la fijación interna rígi$\mathrm{da}^{4}$. Por otro lado, Proffit reportó que durante el primer año posterior a la cirugía, los pacientes tratados con ADF clase II son más estables que los tratados con ADF clase III. Entre uno a cinco años de postoperatorio, algunos pacientes en ambos grupos experimentan ciertos cambios esqueléticos, pero los pacientes con ADF clase III terminan siendo más estables que los pacientes con ADF clase $\mathrm{II}^{1}$.

Algunos de los factores que parecen tener la mayor influencia en la estabilidad esquelética postoperatoria son el movimiento del maxilar o la mandíbula, qué dirección y qué tipo de movimiento se está realizando y la cantidad de movimiento ${ }^{4}$.

La literatura refiere que actualmente se pueden obtener resultados clínicamente satisfactorios y estables a largo plazo en la gran mayoría de los pacientes de cirugía ortognática, sin embargo, hay que tener que cuenta las diferencias entre los distintos tipos de movimiento cuando se realiza la planificación del tratamiento.

En el presente trabajo se evidenció un predominio de ADF clase II en el grupo con rotación del complejo maxilomandibular, lo cual corresponde con el tipo de movimiento con predominio de rotación antihoraria del complejo con el fin de corregir el retrognatismo mandibular y satisfacer las necesidades tanto estéticas como funcionales. Por otro lado, el grupo convencional tuvo un predominio de ADF clase III, los cuales son corregidos con procedimientos convencionales logrando adecuados resultados.

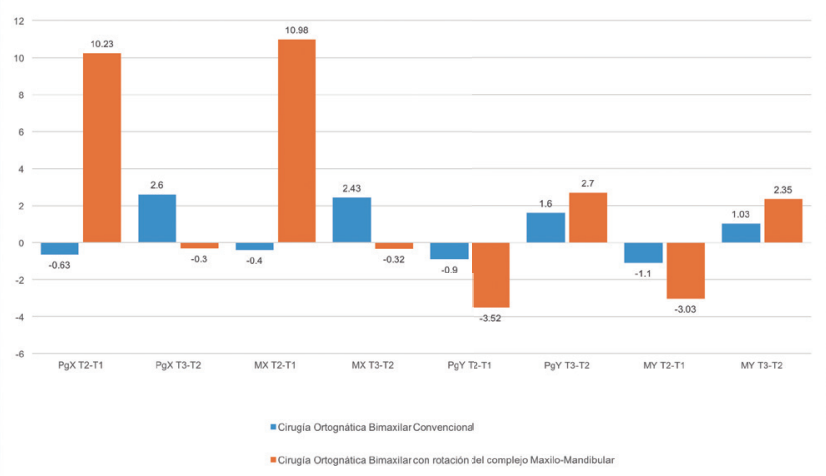

Figura 3. Cambios quirúrgicos y postquirúrgicos de mentoplastia.

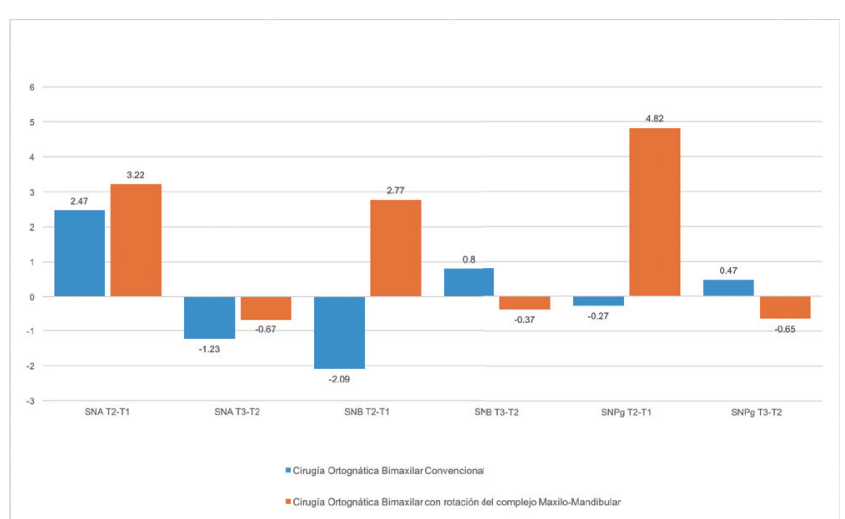

Figura 4. Cambios quirúrgicos y postquirúrgicos de ángulos faciales. 
A pesar de que lo más frecuente es rotación antihoraria en casos con ADF clase II, existen algunos casos de ADF clase III con perfiles faciales hiperdivergentes severos que también se benefician de este tipo de tratamiento.

El relapso o la recidiva de algunos de los movimientos en cirugía ortognática es considerado una circunstancia con la cual los cirujanos orales y maxilofaciales han tenido que lidiar. La tracción muscular y la función, la falta de rigidez en la fijación o el crecimiento posquirúrgico son algunos de los elementos a los cuales se atribuye la recidiva postoperatoria ${ }^{2}$. Ciertos de los factores que parecen tener la mayor influencia en la estabilidad esquelética postoperatoria son el movimiento del maxilar o la mandíbula, la dirección, la cantidad y el tipo de movimiento llevado a cabo ${ }^{4}$.

Los procedimientos de cirugía ortognática han sido clasificados de acuerdo a la estabilidad postoperatoria en muy estable, estable, estable con fijación rígida y problemática ${ }^{1,5}$. Para una estabilidad aceptable, la fijación interna rígida (FIR) no parece ser necesaria en los procedimientos de las categorías muy estables o estables. La FIR hace una diferencia, sin embargo, cuando se realizan movimientos bimaxilares ${ }^{5}$, es por esto que todas las osteotomías realizadas en nuestra población fueron fijadas internamente con material de osteosíntesis.

La estabilización de la OSRMB cuenta con diversas técnicas y formas de fijación interna rígida. Con respecto a la estabilidad de la misma en los movimientos de retroceso mandibular, Al-Moraissi y Ellis ${ }^{6}$ llevaron a cabo un metanálisis en el cual concluyen que no existen diferencias estadísticamente significativas entre la fijación interna con tornillos bicorticales us. miniplaca con tornillos monocorticales. Por otro lado, cuando se lleva a cabo movimiento de avance mandibular, Al-Moraissi y Al-Hendi ${ }^{7}$ llevaron a cabo un metanálisis en el cual encontraron resultados similares sin cambios estadísticamente significativos entre ambas formas de fijación interna. En nuestro estudio, todos los pacientes fueron sometidos a OSRMB estabilizadas con fijación interna, la mayoría de los casos con tornillos bicorticales seguido de la utilización de miniplaca con tornillos monocorticales.

Proffit y cols. consideran cambios postoperatorios $<2 \mathrm{~mm}$ dentro del rango del error del método y clínicamente insignificantes; de 2-4 mm fuera del intervalo de error del método y potencialmente clínicamente significativos y $>4 \mathrm{~mm}$, a menudo más allá del rango de compensación ortodrómica y clínicamente altamente significativos ${ }^{5}$. En nuestro trabajo se evidenció el cambio postoperatorio mayor en el grupo de cirugía ortognática convencional en el punto BX $(2,77 \mathrm{~mm})$, lo cual corresponde a un cambio clínico significativo potencial, ninguna de las medidas tuvieron cambios considerados como clínicamente altamente significativos.

Con respecto a la estabilidad de los resultados en rotación del complejo maxilomandibular, en particular con la rotación en sentido antihorario, a menudo han sido altamente cuestionados ${ }^{8,9}$

El tiempo de seguimiento postoperatorio es considerado un factor importante en la evaluación de la estabilidad esquelética, ya que se ha reportado que los cambios clínicamente relevantes (más de $2 \mathrm{~mm}$ ) ocurren en un porcentaje sorprendentemente alto de pacientes de cirugía ortognática de uno a cinco años después del tratamiento, después de finalizada la cicatrización quirúrgica ${ }^{5}$. El tiempo postoperatorio promedio en nuestro estudio fue de 19,8 meses en el grupo I y de 20,1 meses en el grupo II (Tabla I), superior al tiempo reportado por la mayoría de los autores en la literatura ${ }^{10-12}$.

La estabilidad esquelética es considerada mayor cuando el movimiento favorece la relajación de los tejidos blandos durante la cirugía y menor cuando hay un estiramiento de los mismos. El reposicionamiento superior o ascenso del maxilar relaja el tejido blando y, por ende, ha demostrado tener una buena estabilidad postoperatoria, sin embargo, el avance mandibular produce un estiramiento de los tejidos blandos y ha demostrado no ser tan estable. Para lograr una excelente estabilidad postoperatoria se requiere de una adecuada adaptación neuromuscular más que simplemente una adaptación muscular ${ }^{5}$.

En el grupo II reportado en nuestro estudio la mayoría de las rotaciones antihorarias fueron a expensas de impactación anterior maxilar, lo cual relaja el tejido blando; sin embargo, logrando grandes avances mandibulares, a pesar de esto no se evidenció cambios significativos en el postoperatorio.

Chemello y cols. demostraron que la disminución del plano oclusal y el avance mandibular es un procedimiento muy estable (AX -0,5 mm; BX 0,3 mm, AY -0,3 mm) ${ }^{13}$ similar a lo reportado en nuestro estudio ( $A X 0,05 \mathrm{~mm}, \mathrm{BX} 0,5 \mathrm{~mm}, \mathrm{AY}$ $1,65 \mathrm{~mm}$ ), ambos sin cambios estadísticamente significativos.

Este estudio demostró que la cirugía ortognática bimaxilar convencional o cirugía ortognática bimaxilar con rotación del complejo maxilomandibular son procedimientos estables sin diferencia estadísticamente significativa entre los dos grupos en referencia a cambios antero-posteriores, verticales y cambios en ángulos faciales a largo plazo. Solo se evidenció diferencia estadísticamente significativa en las medidas de BX, PgX, MX, sin embargo, llama la atención que esto se produjo como consecuencia de mayores cambios postoperatorios en el grupo de cirugía ortognática bimaxilar convencional (Tabla IV), contrario a lo reportado en la literatura, en donde el mayor cambio se evidenció en los grupos de rotación del complejo maxilomandibular ${ }^{10}$.

En un metanálisis publicado por Al-Moraissi y Wolford ${ }^{14}$, donde evaluaron 133 pacientes incluidos en tres estudios, concluyen que no hubo diferencia estadísticamente significativa entre rotación horaria us. antihoraria del complejo maxilomandibular para la evaluación de la estabilidad esquelética entre el postoperatorio inmediato y el seguimiento tardío en relación con las medidas verticales y horizontales en el punto A y punto $\mathrm{B}(p=>0,05)^{14}$.

Diversos autores han reportado la importancia de la condición de la ATM previo al procedimiento quirúrgico de cirugía ortognática bimaxilar y consideran un factor determinante ${ }^{13,14}$, sin embargo, Gonçalves y cols. ${ }^{15}$ reportan que incluso el avance maxilomandibular con rotación en sentido contrario a las agujas del reloj del plano oclusal es estable para pacientes sometidos a un reposicionamiento simultáneo del disco articular, usando la técnica de anclaje Mitek. Aquellos pacientes con desplazamiento del disco articular que se sometieron a cirugía ortognática bimaxilar sin ninguna intervención de la ATM experimentaron una recaída significativa ${ }^{15}$. En nuestra población la ATM no fue evaluada de manera sistemática, sin embargo se encontraron resultados estables en el tiempo (seguimiento postoperatorios promedios de 20 meses [DE $\pm 13,3]$ ). 


\section{Conclusiones}

En la actualidad se considera a la cirugía ortognática con rotación del complejo maxilomandibular, tanto en sentido horario como antihorario, como un procedimiento estable cuando se utiliza fijación interna rígida, cuando se está en presencia de ATM sanas y cuando la rotación se lleva a cabo en un punto a través del cóndilo mandibular ${ }^{10,11,13,14}$.

El presente estudio se trató de una pequeña muestra no aleatoria, por lo que se recomienda realización de nuevos estudios aleatorizados con cálculo de tamaño de muestra con el fin de determinar si existe diferencia significativa entre los grupos y cuáles son los factores que influyen en la misma.

\section{Conflicto de intereses}

Los autores declaran no tener ningún conflicto de intereses.

\section{Fuente de financiación}

Los autores niegan alguna relación financiera ni personal que pueda suponer un conflicto de interés.

\section{B I B L I O G R A F Í A}

1. Bailey L', Cevidanes LHS, Proffit WR. Stability and predictability of orthognathic surgery. Am J Orthod Dentofacial Orthop. 2004;126(3):273-7. DOI: 10.1016/S0889540604005207.

2. Serafin B, Perciaccante VJ, Cunningham LL. Stability of orthognathic surgery and distraction osteogenesis: options and alternatives. Oral Maxillofac Surg Clin North Am. 2007;19(3):31120, v. DOI: 10.1016/j.coms.2007.05.002.

3. Hunsuck EE. A modified intraoral sagittal splitting technic for correction of mandibular prognathism. J Oral Surg. 1968;26(4):250-3.

4. Van Sickels JE, Richardson DA. Stability of orthognathic surgery: a review of rigid fixation. Br J Oral Maxillofac Surg. 1996;34(4):279-85.

5. Proffit WR, Turvey TA, Phillips C. The hierarchy of stability and predictability in orthognathic surgery with rigid fixation: an update and extension. Head Face Med. 2007;3:21. DOI: 10. 1186/1746-160X-3-21.
6. Al-Moraissi EAM, Ellis E. Stability of bicortical screw versus plate fixation after mandibular setback with the bilateral sagittal split osteotomy: A systematic review and meta-analysis. Vol. 45, International Journal of Oral and Maxillofacial Surgery. 2016;45(1):1-7. DOI: 10.1016/j.ijom.2015.09.017.

7. Al-Moraissi EA, Al-Hendi EA. Are bicortical screw and plate osteosynthesis techniques equal in providing skeletal stability with the bilateral sagittal split osteotomy when used for mandibular advancement surgery? A systematic review and meta-analysis. Vol. 45, International Journal of Oral and Maxillofacial Surgery. 2016;45(1):1-7. DOI: 10.1016/j. ijom.2015.09.017.

8. Wolford LM, Chemello PD, Hilliard F. Occlusal plane alteration in orthognathic surgery-part I: Effects on function and esthetics. Am J Orthod Dentofac Orthop. 1994;106(3):304-16. DOI: 10.1016/S0889-5406(94)70051-6

9. Posnick JC, Fantuzzo JJ, Orchin JD. Deliberate Operative Rotation of the Maxillo-Mandibular Complex to Alter the A-Point to B-Point Relationship for Enhanced Facial Esthetics. J Oral Maxillofac Surg. 2006;64(11):1687-95. DOI: 10.1016/j. joms.2005.11.118.

10. Reyneke JP, Bryant RS, Suuronen R, Becker PJ. Postoperative skeletal stability following clockwise and counter-clockwise rotation of the maxillomandibular complex compared to conventional orthognathic treatment. Br J Oral Maxillofac Surg. 2007;45(1):56-64. DOI: 10.1016/j.bjoms.2005.12.015.

11. Bang S-M, Kwon Y-D, Kim S-J, Lee B-S, Choi B-J, Ohe J-Y, et al. Postoperative Stability of 2-Jaw Surgery With Clockwise Rotation of the Occlusal Plane. J Craniofac Surg. 2012;23(2):486-90. DOI: 10.1097/SCS.0b013e3182413f6b.

12. Rocha VÁDC, Neto AIT, Rebello IMCR, De Souza GMM, Esteves LS, Santos JN Dos, et al. Skeletal stability in orthognathic surgery: Evaluation of methods of rigid internal fixation after counterclockwise rotation in patients with class II deformities. Br J Oral Maxillofac Surg. 2015;53(8):730-5. DOI: 10.1016/j. bjoms.2015.05.002.

13. Chemello PD, Wolford LM, Buschang PH. Occlusal plane alteration in orthognathic surgery-Part II: Long-term stability of results. Am J Orthod Dentofac Orthop. 1994;106(4):434-40. DOI: 10.1016/S0889-5406(94)70066-4.

14. Al-Moraissi EA, Wolford LM. Is Counterclockwise Rotation of the Maxillomandibular Complex Stable Compared With Clockwise Rotation in the Correction of Dentofacial Deformities? A Systematic Review and Meta-Analysis. J Oral Maxillofac Surg. 2016;74(10):2066.e1-2066.e12. DOI: 10.1016/j. joms.2016.06.001.

15. Gonçalves JR, Cassano DS, Wolford LM, Santos-Pinto A, Márquez IM. Postsurgical Stability of Counterclockwise Maxillomandibular Advancement Surgery: Affect of Articular Disc Repositioning. J Oral Maxillofac Surg. 2008;66(4):724-38. DOI: 10.1016/j.joms.2007.11.007. 